\title{
Convinzioni degli insegnanti di scuola elementare e problemi matematici
}

\author{
Beliefs of primary school teachers \\ and mathematical problems
}

\author{
Annarita Monaco \\ NRD di Bologna, Università degli Studi di Roma “La Sapienza” - Italia
}

Sunto / L'articolo tratta delle convinzioni degli insegnanti di scuola elementare ${ }^{1}$ riguardo ai problemi matematici.

I partecipanti alla ricerca sono stati 45 insegnanti di scuola elementare ai quali è stata sottoposta una lunga intervista al fine di indagare: le convinzioni dei docenti riguardo ai problemi matematici e al proprio ruolo nel gestirne la pratica didattica, le abitudini nell'implementare le raccomandazioni promosse dalle Indicazioni Nazionali e l'analisi di alcuni concetti chiave presenti nella premessa al documento. L'analisi qualitativa del contenuto delle interviste è stata effettuata con l'aiuto del software NVIVO 11. In questo articolo si esaminano i risultati relativi al primo aspetto indagato nelle interviste.

Parole chiave: convinzioni; rappresentazioni; problem solving; metacognizione; formazione docenti.

\begin{abstract}
The article deals with the beliefs of primary school teachers on mathematical problems. The participants in the research were 45 primary school teachers who were given a long interview in order to investigate: the teachers' convictions about mathematical problems and their role in managing the relative teaching practice, the way teachers implement the recommendations of the National Guidelines and the analysis of some key concepts occurring in the introduction to the document. The qualitative analysis of the content of the interviews was carried out with the help of the NVIVO 11 software. This article examines the results related to the first aspect investigated in the interview.
\end{abstract}

Keywords: beliefs; representations; problem solving; metacognition; teacher training.

Nelle ore in cui viene proposta la matematica nella scuola elementare, molto tempo è dedicato alla risoluzione di esercizi scritti, utili per consolidare e verificare l'acquisizione e la padronanza di regole e tecniche apprese in aula. Le proposte dei cosiddetti "problemi" sono spesso null'altro che esercizi (D'Amore, 2014) e mettono in gioco ben poco le componenti noetiche, strategiche, comunicative e semiotiche dell'apprendimento matematico; nella maggior parte dei casi ci si concentra al massimo su componenti algoritmiche (Fandiño Pinilla, 2008).

I libri di testo spesso includono numerose pagine contenenti "problemi" (in realtà esercizi, come vedremo di seguito) già suddivisi in: problemi di addizione, di sottrazione, di calcolo delle frazioni, senza soluzione o impossibili ecc., per affrontare i quali in realtà non appare necessario alcun atto strategico o creativo dell'alunno, essendo già stato implicitamente suggerito il procedimento risolutivo.

Ora, nella parte della premessa alla sezione "Matematica" delle Indicazioni Nazionali italiane (MIUR, 2012, p. 60) si legge:

1. La ricerca presentata in questo articolo è stata condotta in Italia con docenti di scuola primaria, che corrisponde alla scuola elementare in Canton Ticino. 


\begin{abstract}
«Caratteristica della pratica matematica è la risoluzione di problemi, che devono essere intesi come questioni autentiche e significative, legate alla vita quotidiana, e non solo esercizi a carattere ripetitivo o quesiti ai quali si risponde semplicemente ricordando una definizione o una regola. Gradualmente, stimolato dalla guida dell'insegnante e dalla discussione con i pari, I'alunno imparerà ad affrontare con fiducia e determinazione situazioni problematiche, rappresentandole in diversi modi, conducendo le esplorazioni opportune, dedicando il tempo necessario alla precisa individuazione di ciò che è noto e di ciò che s'intende trovare, congetturando soluzioni e risultati, individuando possibili strategie risolutive».
\end{abstract}

Il brano è ricco di spunti fondamentali per la riflessione dei docenti, utilissimi per orientare le scelte rispetto alle caratteristiche dei tipi di problemi da proporre agli alunni e alle metodologie e strategie da utilizzare nel corso delle attività in aula: far discutere, argomentare, proporre contesti autentici e significativi legati alla vita quotidiana, valorizzare le strategie e le rappresentazioni spontanee degli allievi, dare tempo per pensare e realizzare processi di pensiero.

Nelle aule scolastiche è ancora poco presente un ambiente di apprendimento che privilegi attività di risoluzione di problemi significativi, intendendo con questo termine problemi che permettono ai bambini di mettersi in gioco con pensieri, parole, personali rappresentazioni e che consentono loro di venire coinvolti in discussioni attive e costruttive, alla ricerca di argomentazioni che giustifichino le loro scelte. D'altra parte, le difficoltà che i docenti incontrano nel corso del loro lavoro, in matematica in generale e nei problemi nello specifico, sono svariate: il timore di non riuscire a trasmettere i numerosi contenuti previsti nel curricolo di matematica, la preoccupazione di non riuscire ad aiutare bambini che non sanno o si rifiutano di risolvere un problema, soprattutto quando esso richiede la messa in gioco di capacità strategiche e creative. Gli insegnanti spesso si chiedono: "Come possiamo fare ad insegnare le strategie utili per poter risolvere i diversi problemi? Si possono insegnare le strategie? Se sì, quali sono le strategie migliori? E se gli alunni ne inventano di nuove, come fare per seguirle e verificarle, dal momento che non sempre possiedono competenze matematiche specifiche?». Le risposte a tali domande sono complesse e non univoche. Accade a volte che gli insegnanti propongano ai loro alunni problemi che ritengono gestibili sia da loro che dagli allievi; in tal modo escludono quei problemi che richiederebbero ai bambini di sperimentare "al buio", forse perché tale situazione può attivare in loro stessi timori e insicurezze. Ripartire dai valori, dalle credenze, dalle emozioni e dai timori degli insegnanti di matematica di scuola elementare sembra essere una scelta obbligata; solo così si possono individuare ed esplicitare quegli elementi cognitivi e affettivi che impediscono ai docenti di avviare una pratica didattica dei problemi profonda ed efficace.

\title{
Focus sui problemi
}

\subsection{Problemi ed esercizi}

Come già anticipato, a scuola (e soprattutto nei libri di testo) spesso sono denominati "problemi" dei compiti che in realtà sono esercizi. Approfondiamo la differenza tra questi due termini, utilizzando le parole di D'Amore e Fandiño Pinilla: 
«[...] Entrambi concernono situazioni problematiche causate da vari fattori: una proposta dell'insegnante (più o meno motivata), test o quiz, effettiva e reale situazione nella quale l'alunno o la classe si ritrova... Ma gli esercizi possono essere risolti utilizzando regole o nozioni già apprese o in via di consolidamento e quindi rientrano nelle categorie "rafforzamento o verifica"; i problemi coinvolgono o l'uso di regole o nozioni (alcune anche in via di esplicitazione proprio in quell'occasione) o la successione di operazioni la cui scelta è atto strategico, talvolta creativo, dell'allievo stesso».

(D’Amore \& Fandiño Pinilla, 2006, p. 647)

In linea con queste definizioni, potremmo dire che l'esercizio trova il proprio spazio cognitivo naturale nella zona di sviluppo effettiva descritta da Vygotskij, mentre il problema nella zona di sviluppo prossimale (Fandiño Pinilla, 2008). Negli esercizi i bambini attivano un procedimento puramente esecutivo; nei problemi è necessario un comportamento strategico e si devono prendere continuamente decisioni, mettendo in gioco, eventualmente, anche regole apprese e automatismi consolidati attraverso gli esercizi. Esercizi e problemi rappresentano attività complementari per la maturazione delle competenze matematiche.

\subsection{Il problem solving}

Le ore di matematica in aula, come si è già sostenuto in precedenza, sono ricche di attività attinenti l'apprendimento di regole, tecniche, simboli e formule che gli alunni devono apprendere e successivamente applicare. Una volta che ha acquisito alcune regole, l'essere umano può usarle per molti scopi, tipicamente nei rapporti con l'ambiente che lo circonda. Può fare anche qualcosa di più importante: può pensare. Ciò significa che egli è in grado di combinare fra loro gli elementi che ha già appreso, e comporre con quelli una grande varietà di regole di ordine superiore. Per fare ciò stimola sé stesso e risponde a vari tipi di sollecitazione da parte dell'ambiente. Grazie al processo di combinazione di regole vecchie in regole nuove, egli risolve problemi che sono nuovi per lui, acquistando così un patrimonio di nuove capacità (D'Amore, 2014). Quindi l'essere umano, nel nostro caso l'allievo, con gli strumenti acquisiti si pone naturalmente nuovi problemi e tenta di risolverli.

I primi studi sistematici sui metodi risolutivi nel contesto matematico risalgono a George Polya, che si pone un obiettivo didattico: insegnare a risolvere problemi. Egli assume come modello di "bravo risolutore" il matematico impegnato nell'attività di ricerca; analizza quindi i processi messi in atto da questi bravi risolutori, tentando di evidenziare quali metodi risolutivi vengono impiegati, nella convinzione di poterli insegnare.

Polya distingue 4 fasi, che sono a suo parere tipiche di ogni processo risolutivo:

- fase 1: si deve comprendere il problema;

- fase 2: si devono scoprire i legami che intercedono tra le varie informazioni, fra ciò che si cerca e i dati, per compilare un piano di risoluzione;

- fase 3: si procede allo sviluppo del piano;

- fase 4: si esamina il risultato e si procede alla sua verifica.

Il bravo risolutore, secondo Polya, si pone in modo naturale alcune domande che stimolano le operazioni mentali utili per la risoluzione e suggeriscono euristiche; tali domande costituiscono lo schema di risoluzione. In questo senso, sembra che le parole di Polya vogliano precorrere l'annuncio di alcune implicazioni didattiche di 
stampo costruttivista dell'apprendimento. La sua idea generale è infatti che l'insegnante, esplicitando queste domande in modo opportuno, possa localmente aiutare lo studente a risolvere uno specifico problema, e più in generale educarlo a porsi tali domande. Polya scrive:

\begin{abstract}
«Un insegnante di matematica ha una grande possibilità. Ovviamente, se egli impiegherà le sue ore di lezione a far eseguire dei calcoli ai suoi studenti, finirà per soffocare il loro interesse, arrestare il loro sviluppo mentale e sciupare I'opportunità che gli si presenta. Invece, se risveglierà la curiosità degli alunni proponendo problemi di difficoltà proporzionate alle conoscenze della scolaresca e li aiuterà a risolvere le questioni proposte con domande opportune, egli saprà ispirare in loro il gusto di un ragionamento originale».
\end{abstract}

(Polya, 1945/2016, p. 7)

È accaduto, però, che i programmi educativi fondati sull'insegnamento di un repertorio di euristiche specifiche si siano rivelati fallimentari: «Gli studenti addestrati in questo modo non riuscivano a generalizzare e a trasferire le conoscenze apprese in altre situazioni» (Zan, 2010, p. 156).

Tali fallimenti aprirono il campo, agli inizi degli anni ' 80 del secolo scorso, a nuovi studi in didattica della matematica che, pur nel riconoscimento del grande contributo dato dagli studi di Polya, ne svilupparono un'analisi critica, mettendo in discussione I'idea che possa esistere un metodo addestrativo per insegnare a risolvere i problemi. In particolare sono stati messi in discussione i due assunti fondamentali da cui era partito Polya: il fatto che per essere un buon solutore di problemi sia sufficiente possedere un buon bagaglio di conoscenze ed euristiche, e l'efficacia dell'insegnamento delle euristiche (Baccaglini Frank, Di Martino, Natalini \& Rosolini, 2018).

Schoenfeld (1985) sottolinea come Polya abbia descritto accuratamente il processo di risoluzione di un problema, ma aggiunge che ciò non è trasferibile a livello didattico, in quanto non fornisce elementi per l'uso efficiente delle strategie. Schoenfeld, nelle sue ricerche, osserva e ascolta diversi studenti alle prese con il problem solving, chiedendo loro di ragionare a voce alta e registra i tempi che essi dedicano alle diverse fasi di risoluzione.

I dati raccolti evidenziano che i "cattivi solutori" dedicano quasi tutto il tempo allo sviluppo di un piano (esplorazione). I "bravi solutori" dedicano molto tempo a riflettere sugli stati di avanzamento dei loro tentativi e saltano da una fase all'altra della risoluzione di un problema sulla base di queste riflessioni. Dunque ciò che caratterizza i buoni risolutori è la quantità e la qualità delle decisioni prese nel corso del processo risolutivo; in questo senso, un ruolo chiave è da attribuire alle competenze di natura metacognitiva, ossia alle capacità di valutare e prendere consapevolezza delle proprie risorse, di prendere decisioni efficienti, in base a tale consapevolezza. I bravi risolutori spendono la maggior parte del tempo a pensare piuttosto che a fare, ponendosi vari tipi di domande, ad esempio: «Che cosa sto facendo?». II bravo risolutore considera più approcci, dei quali molti sbagliati, ma non li porta mai fino in fondo perché: «[...] è tanto inesorabile nel controllare e rifiutare idee, quanto ingegnoso nel generarle» (Schoenfeld, 1987, p. 194).

Schoenfeld propone di dividere il comportamento risolutivo in vari "episodi": 1. lettura; 2. analisi; 3. esplorazione; 4. pianificazione; 5. implementazione; 6. verifica; 7. transizione. In questi episodi si possono riconoscere le quattro fasi di Polya.

Volendo ragionare sulle differenze, possiamo notare la sottile distinzione tra analisi 
ed esplorazione (che in Polya sono comprese nella fase di comprensione) e nell'introduzione dell'episodio di transizione.

Le ricerche di Schoenfeld sono esemplificative di come la ricerca, già tre decenni fa, avesse sottolineato la complessità del processo di risoluzione dei problemi, identificando il ruolo di fattori diversi, oltre alle conoscenze e alle euristiche (Baccaglini Frank et al., 2018).

È stata molte volte sottolineata in letteratura la significatività del promuovere un approccio per problemi a livello di educazione matematica di base, per diversi motivi: garantire una buona formazione matematica, sulla base della convinzione che il problem solving abbia in sé «il potenziale per stimolare sfide intellettuali che possano favorire lo sviluppo matematico dello studente» (NCTM, 2010); un secondo aspetto è legato alla convinzione che affrontare problemi sia l'attività potenzialmente più affascinante del fare matematica; infine un terzo aspetto è legato al contributo che l'educazione matematica può dare per la formazione del cittadino adulto (Baccaglini Frank et al., 2018).

\section{Focus sulle convinzioni}

\subsection{Il costrutto di convinzione}

Il termine convinzione (o credenza), traduzione dall'inglese belief, è mutuato dalla psicologia sociale (Rokeach, 1960). D'Amore e Fandiño Pinilla hanno assunto la seguente definizione di convinzione:

«convinzione (belief) (o credenza): opinione, insieme di giudizi/attese, quel che si pensa a proposito di qualcosa; I'insieme delle convinzioni di qualcuno (A) su qualcosa $(T)$ dà la concezione $(K)$ di A relativamente a $T$; se $A$ appartiene ad un gruppo sociale (S) e condivide con gli altri appartenenti ad S quell'insieme di convinzioni relativamente $\mathrm{a} \mathrm{T}$, allora $\mathrm{K}$ è la concezione di $\mathrm{S}$ relativamente $\mathrm{a} \mathrm{T}$ ».

(D’Amore \& Fandiño Pinilla, 2004, p. 28)

Come sostengono Furinghetti e Pehkonen (2002), l'interesse per le convinzioni e i sistemi di convinzioni emerge principalmente negli anni '70, attraverso gli sviluppi nelle scienze cognitive (Abelson, 1979). L'assunto di base è che gli individui ricevano continuamente segnali dal mondo che li circonda. Secondo le percezioni ed esperienze basate su questi messaggi, gli individui traggono conclusioni su diversi fenomeni e sulla loro natura. La conoscenza soggettiva degli individui, cioè le loro convinzioni (compresi i fattori affettivi) sono una composizione di queste conclusioni. Le convinzioni, che gli individui confrontano con nuove esperienze e con le convinzioni di altri individui, sono continuamente valutate e si possono modificare. Quando viene adottata una nuova convinzione, questa farà automaticamente parte della struttura più ampia della conoscenza soggettiva degli individui, cioè del loro sistema di convinzioni. Tale sistema di convinzioni di un individuo è composto dalle sue convinzioni consce o inconsce, ipotesi o aspettative e dalle loro combinazioni (Green, 1971).

Pian piano l'accezione data al termine "beliefs" si allarga. McLeod (1985, p. 268) è uno dei primi ricercatori a sottolineare la necessità di sistemare teoricamente il concetto di convinzione in matematica: «I sistemi di convinzioni possono essere applicati 
al contenuto matematico, per esempio, o all'idea che un individuo ha delle proprie possibilità di successo nel risolvere un problema».

McLeod $(1989,1992)$ cerca di inquadrare la ricerca sul tema e di costruire una teoria intorno al concetto di convinzione, interpretando nuovi fatti e partendo dall'assunto che le convinzioni giochino un ruolo fondamentale nei processi conoscitivi. Ogni persona ha nel suo sistema di convinzioni una struttura che possiamo quindi definire quasi-logica, nel senso che ci sono alcune "convinzioni primarie" e altre "derivate". Può capitare che in una persona, con il passare del tempo, due convinzioni si scambino i ruoli: ciò che prima era primario diventa derivato e viceversa. Questa dimensione quasi-logica può avere delle importanti conseguenze nel momento in cui si tenta di modificare una convinzione. Se ci si pone come obiettivo quello di modificare una convinzione $A$, che nel sistema di convinzioni dell'individuo è derivata, è chiaro che risulteranno poco efficaci gli interventi che non si preoccupino di incidere sulla convinzione primaria da cui la convinzione A deriva.

Studiare le convinzioni e la loro organizzazione in sistemi può aiutare a superare un'ambiguità spesso riscontrata in didattica della matematica: la contraddizione tra le convinzioni che un soggetto dichiara (ad esempio quando risponde a un questionario o a un'intervista) e quelle che invece "pratica", ossia quelle che guidano i suoi processi decisionali (Zan, 2010).

\subsection{Insegnanti, insegnamento e convinzioni}

Il concetto di convinzione fa riferimento dunque a un costrutto mentale di un individuo (o di un gruppo sociale, nel caso si tratti di convinzione diffusa), che si forma in relazione a ciò che il soggetto già conosce e alle nuove esperienze con l'ambiente. Richardson (1996) mette in luce soprattutto questo concetto di "beliefs", da intendersi come quelle convinzioni che interagiscono con la prassi e ne influenzano il cambiamento.

Tali convinzioni sono importanti elementi predittivi delle pratiche professionali e richiedono una particolare attenzione nel momento in cui si vuole mettere in atto un qualsiasi processo di cambiamento e di innovazione, in quanto agendo su tali convinzioni possiamo indirettamente agire sui comportamenti. Molteplici studi, inoltre, hanno verificato la relazione inversa: la modifica della pratica può intervenire ed agire sul cambiamento di percezioni, convinzioni, atteggiamenti (ad esempio, si veda Vannini, 2012). L'essere umano si costruisce sulla base delle esperienze quotidiane e delle concettualizzazioni che si organizzano in insiemi coerenti e sistematici che le rendono difficilmente modificabili con la sola aggiunta di informazioni o fatti. D'altra parte, perché il cambiamento possa avvenire, è necessaria una modificazione profonda della teoria esistente (Carey, 1999; Vosniadou, 1994). Ci si può interrogare su quando e come si strutturano le convinzioni degli insegnanti sull'insegnamento, su quali sono le teorie implicite sulla scuola e sull'educazione e su quali siano i processi che possono permettere la loro messa in crisi, per poter modificare i comportamenti dell'insegnante (Vannini, 2012).

Esplicitare le convinzioni latenti in contesti di formazione iniziale e continua degli insegnanti sembra essere la via maestra da percorrere. Molti studi sull'insegnamento e sulla formazione dei docenti degli ultimi anni hanno affrontato tale tematica; in questo panorama ha assunto interesse il concetto di "riflessività" applicata alla professionalità dei docenti, che fa riferimento alla prospettiva deweyana sull' "insegnante riflessivo" da una parte e alle ipotesi teoriche di Schön (1983) sull'apprendimento 
degli adulti dall'altra. Si dà valore all'accompagnamento degli insegnanti in percorsi di progressiva assunzione di consapevolezza, a partire dal senso comune, fino ad arrivare a una competenza professionale sempre più piena. La formazione degli insegnanti (sia iniziale che continua) dovrebbe sempre partire dal fare esplicitare ai docenti le proprie convinzioni, al fine di poter poi agire su di esse attraverso azioni che favoriscano processi di razionalità, che siano adatti ad analizzare e a mettere in crisi convinzioni connesse a pratiche inefficaci, per poter costruire nuove convinzioni e atteggiamenti pedagogicamente fondati. Entro tali processi di ristrutturazione un ruolo importante è ricoperto dai saperi disciplinari, nel nostro caso il sapere matematico: proposto in modo scientificamente fondato, e anche credibile, tale sapere può mettere in crisi quelle convinzioni dei docenti che ostacolano l'attivazione di processi cognitivi e di approfondimento personale, oltre ogni banale accumulazione (D'Amore \& Fandiño Pinilla, 2009).

\subsection{Le convinzioni sui problemi matematici: la ricerca internazionale}

La risoluzione dei problemi è una competenza chiave e un mezzo importante per lo sviluppo di altre competenze matematiche e non matematiche. Nonostante ciò, nella maggior parte dei programmi scolastici la sua inclusione rimane poco chiara e nei repertori professionali degli insegnanti essa risulta poco presente (Andrews \& Xenofontos, 2014).

La ricerca sulle convinzioni degli insegnanti sulla natura dei problemi matematici indica sostanziali variazioni a livello internazionale: Cai e Nie (2007) hanno trovato differenze significative tra insegnanti cinesi e insegnanti americani, derivanti da loro esperienze come studenti di matematica. Andrews (2007) ha intervistato insegnanti ungheresi che hanno descritto la matematica come una materia che si basa su una sfida intellettuale intorno ai problemi. Altri ricercatori hanno identificato differenze sostanziali, culturalmente costruite, nelle convinzioni di insegnanti di scuola elementare in formazione iniziale a Cipro e in Inghilterra (Xenofontos \& Andrews 2012, 2014). Le convinzioni degli insegnanti sui problemi e sulla risoluzione dei problemi, quindi, sono legate alle culture di appartenenza; la realtà della vita di classe ha un effetto moderatore sulle convinzioni degli insegnanti intorno alla risoluzione dei problemi. Per alcuni insegnanti, in particolare per i neo-assunti, tale realtà può compromettere il rafforzamento delle credenze relative al ruolo e al primato del problem solving nelle classi di matematica. Altri insegnanti, per attuare le convinzioni appena acquisite sui problemi e sulla risoluzione dei problemi, abbandonano le pratiche di classe esistenti, magari inadeguate, a favore di pedagogie più aperte (Andrews \& Xenofontos, 2014). Nel panorama delle ricerche effettuate a livello internazionale sul tema, ai fini del presente lavoro risulta particolarmente interessante la ricerca di Andrews e Xenofontos (2014); nella loro ricerca, i due studiosi indagano convinzioni di tre insegnanti ciprioti di scuola elementare riguardo ai problemi e alla loro risoluzione, ma anche la loro competenza come risolutori di problemi matematici e gli aspetti del relativo insegnamento. Tale studio ha non solo l'obiettivo di esaminare le interazioni tra convinzioni, competenze e pratica degli insegnanti, ma anche quello di mettere a punto un kit di strumenti appropriato per questa tipologia di studi. Gli autori ritengono che il loro specifico oggetto di ricerca possa essere meglio affrontato con uno studio di caso multiplo. Viene utilizzato un quadro teorico che tiene conto sia delle ricerche di Schoenfeld (1992, 2004), negli Stati Uniti, che delle ricerche di De Corte (1995, 2004), Verschaffel e altri nelle Fiandre (De Corte, Verschaffel, Op't Eynde, 2000). 
Basandosi su questi quadri teorici, Andrews e Xenofontos (2014) costruiscono uno strumento che gli permette di acquisire narrazioni dettagliate per ciascun insegnante; in ciascuno scritto è evidente la complessa attività di relazione tra il modo in cui gli insegnanti pensano e quello in cui agiscono (Cohen, 1990; Skott, 2009; Wilkins, 2008). Dal confronto tra i tre studi di caso, emergono in particolare le convinzioni e le pratiche di una delle insegnanti; tali pratiche sembrano essere coerenti e legate a convinzioni radicate, secondo le quali i problemi sono concepiti come delle routine e dei compiti alla risoluzione dei quali dedicare un tempo limitato. L'analisi approfondita condotta da Andrews e Xenofontos (2014) gli permette di identificare regolarità e irregolarità, e di delineare, anche se in modo provvisorio, una descrizione esauriente di ciascun insegnante coinvolto.

Pehkonen (1993) affronta la problematica da un altro punto di vista, indagando le convinzioni dei formatori degli insegnanti di matematica finlandesi sull'implementazione del problem solving e raccoglie i dati mediante un questionario distribuito a 43 insegnanti nel corso di un seminario di problem solving.

I risultati sono così sintetizzati:

- la risoluzione dei problemi è importante, perché aiuta a favorire la preparazione cognitiva degli alunni;

- I'insegnamento relativo alla risoluzione dei problemi dovrebbe essere effettuato in modo creativo, flessibile e rassicurante;

- gli insegnanti dovrebbero coinvolgere gli alunni nella risoluzione dei problemi, permettendo loro di risolverli;

- la disponibilità degli alunni a studiare il problem solving è il prerequisito più importante per insegnare a risolvere problemi.

Dopo aver indagato il punto di vista dei formatori, Pehkonen (2017) si è quindi proposto di studiare le convinzioni degli insegnanti di scuola elementare finlandesi in materia di problem solving e del suo insegnamento.

Le risposte degli insegnanti, le loro idee sul problem solving e sulla pratica didattica ad esso relativa sono classificate sulla base delle seguenti categorie:

1. il significato del curriculum;

2. il significato dei materiali didattici;

3. I'insegnamento delle capacità di problem solving.

In questa ricerca si evidenzia che ci sono troppi argomenti nel curriculum matematico e c'è anche poco tempo disponibile per attuarlo; c'è fretta di insegnare i troppi argomenti di base e i soggetti più in difficoltà hanno bisogno di tempo e di supporto sugli argomenti di base. Gli insegnanti dichiarano di integrare il lavoro sui problemi con compiti aggiuntivi di problem solving, specialmente nel caso di alunni motivati e di talento; esprimono la convinzione che la risoluzione dei problemi implichi l'applicazione di conoscenze e abilità precedentemente acquisite. Nonostante riconoscano l'importanza del problem solving, gli insegnanti ammettono anche la loro non adeguatezza nell'implementare un insegnamento che corrisponda alle loro convinzioni, a causa della pressione esterna esercitata dal curriculum e dal contesto sociale di cui tale curriculum è l'espressione.

Rispetto all'insegnamento delle capacità di problem solving, dai risultati dello studio emerge che un insegnante dovrebbe: studiare le strategie con le quali il risolutore dovrebbe risolvere nuovi tipi di compiti, cioè i problemi; lasciare che l'alunno selezioni da sé quale strategia usare. Un insegnante, inoltre, dovrebbe modellare e insegnare, passo dopo passo, il pensiero in fasi o insegnare strategie di pensiero, illustrate con diversi modelli oppure ponendosi come leader che illustra, fornisce 
esempi, apre problemi, spiega il proprio pensiero durante il processo di soluzione, fornisce le informazioni sulle relazioni di causa-effetto e su diverse strategie di problem solving; inoltre, i docenti mettono in evidenza che alcune strategie permettono I'avanzamento passo dopo passo nella suddivisione del problema in sottoproblemi. Gli insegnanti pongono l'attenzione anche sulla debolezza delle loro capacità, soprattutto nel momento in cui devono dare consigli agli alunni oppure quando si tratta di riuscire a guidare un alunno in modo tale che sia comunque lui stesso a risolvere il problema. Per alcuni insegnanti è stato utile provare interesse e sperimentare strategie nella risoluzione dei problemi, perché tali elementi li hanno aiutati a cercare e a trovare i propri metodi di insegnamento e i materiali adeguati.

Pehkonen (2017) conclude pertanto che le convinzioni dei docenti sulla risoluzione dei problemi matematici facciano parte di un insieme più ampio che include le convinzioni degli insegnanti sulla natura della matematica e quelle sul suo insegnamento e apprendimento.

\section{La nostra ricerca}

Nel lavoro che si sta presentando si assume che il costrutto di convinzione sia uno strumento teorico utile per poter indagare le relazioni tra docenti, problemi e pratica didattica. Si focalizza l'attenzione su quegli aspetti che Zan (2010) mette in evidenza parlando dei processi di controllo che un soggetto attiva quando prende delle decisioni in un contesto di apprendimento della matematica: le convinzioni che ha sulla matematica (la sua epistemologia personale) e sulle sue teorie del successo; le convinzioni che ha su di sé e in particolare il senso di autoefficacia; un ruolo importante hanno anche gli aspetti motivazionali e, più in generale, gli aspetti affettivi e le emozioni associate alla matematica.

Le categorie citate costituiscono un punto di partenza utile per ridefinire in modo specifico gli obiettivi dello studio delle convinzioni dei docenti relativamente ai problemi.

\subsection{Le domande della ricerca}

II principale interrogativo che ci si pone è il seguente:

"Quali sono le convinzioni degli insegnanti di scuola elementare nei confronti dei problemi matematici, della loro risoluzione e della relativa pratica didattica?»

Tale interrogativo si articola in tre domande di ricerca, ciascuna delle quali è ulteriormente definita attraverso alcune sotto-domande; tali sotto-domande hanno avuto la funzione di guida nella raccolta dei dati e nella loro analisi.

Di seguito si presentano domande e sotto-domande:

D1. Quali sono le convinzioni degli insegnanti di scuola elementare sui problemi matematici?

- Per gli insegnanti, quali caratteristiche deve avere un problema matematico?

- Quale/i tipologia/e di problema/i gli insegnanti apprezzano maggiormente? Per quali ragioni?

- Ci sono delle differenze tra apprezzamento di determinati problemi e una dichia- 
rata disponibilità a proporli in classe? Se sì, quali sono le ragioni?

- Quale valutazione gli insegnanti danno dei problemi presenti sui libri di testo? Come li modificherebbero per renderli buoni problemi?

D2. Quali sono le rappresentazioni che gli insegnanti hanno delle Indicazioni Nazionali per il curricolo rispetto ai problemi e alla loro pratica didattica?

- Per progettare il loro lavoro sui problemi, gli insegnanti tengono conto delle Indicazioni Nazionali? Quali parti utilizzano? Come spiegano le loro risposte?

- Quali concezioni gli insegnanti hanno di alcuni concetti chiave presenti nel testo delle Indicazioni Nazionali: discussione, argomentazione, chiarezza, contesto significativo legato alla vita quotidiana?

D3. Quale ruolo deve avere l'insegnante nella gestione del lavoro didattico sui problemi?

- Quali strategie didattiche predilige un insegnante al fine di rendere più proficuo il lavoro sui problemi matematici con i suoi alunni?

- Quali convinzioni hanno gli insegnanti sugli alunni buoni risolutori? Quali strumenti può avere un insegnante per aiutare un suo alunno a diventare un buon risolutore?

- Quali bisogni formativi ha l'insegnante rispetto ai problemi e alla gestione della loro pratica didattica.

In questo contributo si esamineranno i dati e i risultati relativi alla prima domanda di ricerca.

\subsection{Le scelte metodologiche}

La ricerca condotta, e presentata in questo articolo, si prefigura come esplorativo-qualitativa (Lumbelli, 2006; Vannini, 2009). Si è scelto di utilizzare una traccia di intervista, come strumento per indagare i sistemi di convinzioni degli insegnanti. Tale scelta è dovuta alla considerazione che i docenti, nel rispondere alle domande poste, possano esplicitare esperienze e storie didattiche in maniera ricca e approfondita, con elementi coerenti ma anche contraddittori, e tendano a "cucirli" introducendo nessi percepiti come causali o semplicemente cronologici (Di Martino, 2004). Tali elementi sono indicativi comunque della complessità delle convinzioni, che sono il nostro oggetto di studio. Ė stato costruito un modello di intervista originale semi-strutturata, con domande sia aperte sia chiuse, appositamente redatta per questo lavoro di ricerca. La sua costruzione è avvenuta per gradi: il testo è la versione finale di uno strumento sperimentato in fase di try-out con 30 docenti di Roma e provincia ed è stato molte volte modificato, sia per quanto riguarda le domande, sia rispetto ai sette problemi scelti e sottoposti all'analisi dei docenti. Non tutti gli intervistati hanno mostrato le stesse abilità e disponibilità di introspezione e di verbalizzazione; in generale i 30 insegnanti coinvolti nel try-out hanno dato un grande contributo alla messa a punto dell'intervista, grazie ai loro commenti e alle loro numerose reazioni e riflessioni.

\subsection{Il campione di ricerca}

Per quanto riguarda la scelta del campione, si è fatto ricorso a un "campionamento casuale stratificato proporzionale", sono stati individuati tre strati di collocazione geografica: Sud e Isole, Centro, Nord. Sono stati contattati, direttamente o attraver- 
so le direzioni didattiche, 45 insegnanti di quarta elementare delle seguenti regioni d'Italia: Lazio, Campania, Puglia, Sicilia, Emilia Romagna, Trentino Alto Adige, Piemonte, suddivisi in: 13 insegnanti del Nord, 18 del Centro e 14 del Sud e delle Isole. Per poter acquisire più elementi possibili, è stato formato un campione che avesse una buona variabilità rispetto a: aree geografiche, anzianità di servizio, numero di anni di insegnamento della matematica nella scuola elementare. Si è scelto di intervistare insegnanti di classe quarta, presupponendo che potessero essere i più adatti a immedesimarsi nella situazione di poter proporre, da lì a pochi mesi, sette problemi diversamente classificati e pensati per la classe quinta, ai propri alunni. Un altro elemento importante di variabilità è stata la formazione dei docenti, non intesa come numero di partecipazioni a corsi di aggiornamento, online o in presenza, a convegni o altro, ma come l'insieme di quelle esperienze che abbiano effettivamente modificato o avviato un processo di modifica delle convinzioni degli insegnanti sul tema oggetto di studio. La variabilità delle formazioni è stata supposta e poi verificata con i dati emersi dalle risposte fornite dai docenti alle domande della prima sezione.

\subsection{L'intervista}

L'intervista, nella sua versione finale, è strutturata in tre sezioni (Allegato 1).

La prima sezione mira a raccogliere i dati dei 45 docenti intervistati: scuola di appartenenza, anzianità di servizio, anni di insegnamento nella scuola elementare, anni di insegnamento in classe quinta elementare. Si chiede inoltre ai docenti di narrare brevemente il proprio rapporto con la matematica e in particolare con i problemi, invitandoli a raccontare le esperienze formative ritenute più significative per l'evoluzione della propria pratica didattica relativa ai problemi. Tali informazioni sono state raccolte per verificare che ci fosse una effettiva variabilità tra i diversi livelli di formazione. La sezione termina con la richiesta di esplicitare le caratteristiche auspicate per un problema matematico e la relazione percepita tra le Indicazioni Nazionali del 2012 e la propria pratica didattica relativa ai problemi.

Nella seconda sezione, molto più corposa, sono presentati sette problemi afferenti a diversi ambiti matematici, diversamente classificati dal ricercatore e pensati per la classe quinta elementare. Tale classificazione non è esplicitata ai docenti intervistati. I docenti sono invitati a leggere e analizzare ciascuno dei problemi sulla base di alcune sollecitazioni. Prima di tutto si sonda la disponibilità del docente a proporre ciascuno dei sette problemi agli alunni della loro futura quinta. Si chiede poi di esplorare ed esprimere il potenziale percepito di discussione e di argomentazione che ciascun problema può sollecitare, il livello di significatività e autenticità del suo contesto, la modalità organizzativa ritenuta più idonea per il lavoro in classe, la possibile utilizzazione del problema come strumento matematico: verifica, applicazione e consolidamento oppure strumento per la costruzione dei concetti. Si chiede al docente di proporre eventuali modifiche al testo per migliorarlo. Per ogni domanda posta si chiede di esplicitare le motivazioni delle risposte. Infine si invita a formare due diverse graduatorie: la prima, sulla base dell'apprezzamento personale dei problemi; la seconda, sulla base della loro percepita fattibilità in aula.

La terza sezione, conclusiva, raccoglie i racconti delle difficoltà, delle azioni didattiche e delle emozioni dei docenti inerenti alla trattazione dei problemi, così come sono nate e si sono sviluppate in aula. Si acquisiscono anche scelte dichiarate rispetto alle strategie didattiche ritenute più utili ai fini di una migliore produttività degli alunni nell'atto di risolvere problemi. Si indaga, infine, sulle opinioni che gli inse- 
gnanti hanno dei problemi proposti sui libri di testo e si chiede, infine, di manifestare i propri bisogni formativi, per migliorare la didattica dei problemi.

Le interviste, della durata media di 70 minuti ciascuna, sono state tutte audio-registrate e trascritte. L'approccio scelto per la loro analisi è stato il "content analysis": sono state definite a priori le categorie, alla luce delle quali sono stati estratti degli stralci di testo, poi classificati (Di Martino, 2004). La lettura delle risposte ha suggerito nuove categorie interpretative, che hanno stimolato una nuova lettura (processo a spirale). Per poter effettuare l'analisi del contenuto è stato utilizzato il software NVIVO $11^{2}$.

\subsection{I problemi scelti}

Sono state utilizzate fonti diverse per poter scegliere i problemi, che sono stati poi testati e modificati in fase di try-out al fine di renderli il più possibile rappresentativi di alcune tipologie di testi, ipotizzate sulla base di criteri definiti a posteriori. Si è tenuto conto del fatto che, sui libri di testo e in letteratura, esistono diverse tipologie di testi di problemi e che ciascuna di esse ha un suo ruolo specifico, a volte anche più di uno, ai fini dello sviluppo delle diverse componenti dell'apprendimento matematico degli allievi. Spesso mancano ai problemi alcuni requisiti che rispondono a quelle caratteristiche di autenticità, significatività, aderenza alla realtà così come vorrebbero le Indicazioni Nazionali (Di Martino \& Zan, 2017). Di seguito, si riportano i testi completi e si descrivono le principali caratteristiche di ciascun problema:

Rocco e il suo giardino (problema standard)

Zio Rocco decide di sistemare il giardino della sua casa al mare. Questo giardino è a forma di rettangolo: misura $6 \mathrm{~m}$ di larghezza e 4,5 $\mathrm{m}$ di lunghezza. Lo zio decide di disporre piante con fiori sul suo contorno. Sistemando una piantina ogni 4 metri, quante piantine riesce a sistemare Rocco? Per ciascuna spende $€$ 3,80. Quanto spende in tutto?

È un problema a più operazioni che coinvolge sia l'ambito aritmetico che quello geometrico: è assimilabile a una tipologia di problemi tipica dei libri di testo. Ci sono diversi oggetti matematici in gioco; I'alunno, per risolverlo, applica conoscenze e regole apprese a scuola.

Le monete (problema narrativo)

Piero e Francesco partono per una gita a piedi. Piero mette nel suo zainetto 5 panini e Francesco mette 7 panini nel suo. Lungo la strada incontrano uno sconosciuto, affamato, ma senza provviste. Decidono di mettere in comune i loro panini e mangiano tutti e tre un ugual numero di panini. Al momento di lasciarsi, lo sconosciuto, come ringraziamento per il pane ricevuto, dà 5 monete a Piero e 7 a Francesco. Piero dice: «Ne devi dare solo 3 a me e 9 a Francesco. Infatti anche noi abbiamo mangiato parte dei 12 panini». Francesco dice: «Dal punto di vista della matematica Piero ha ragione. Ma l'importante

2. NVIVO è un ambiente di lavoro che permette l'importazione dei dati acquisiti con le interviste, la loro codifica in categorie e sottocategorie, la loro analisi, visualizzazione e presentazione. 
è che ognuno di noi ha messo quello che aveva: quindi dai 6 monete a Piero e 6 a me». Lo sconosciuto non sa più cosa fare. Non capisce perché dal punto di vista della matematica sarebbe più giusto dare 3 monete a Piero e 9 a Francesco.

Prova a spiegarglielo. Tu al posto dello sconosciuto cosa faresti? 6 monete a Piero e 6 a Francesco? Oppure 3 a Piero e 9 a Francesco? Oppure 5 monete a Piero e 7 a Francesco?

Si tratta di un testo narrativo, che è la formulazione "a righe" ${ }^{3}$ di un problema tratto dal capitolo quarto (Pane e pensiero) dell'Uomo che sapeva contare, (Tahan, Negrin \& Zannini, 1996). Le soluzioni proposte si basano su valutazioni diverse, alcune squisitamente matematiche, altre no. I processi decisionali che portano alla scelta della soluzione sono il frutto di un bilancio tra diversi elementi, in cui entra in gioco la matematica con il ragionamento proporzionale, ma anche valutazioni di carattere morale e affettivo (Zan, 2016).

Una mostra in aula (compito di realtà)

Scegli una parete della tua aula, dove esporre i tuoi lavori più significativi effettuati in classe. Misura le dimensioni della parete. Hai a disposizione cartelloni di misura $70 \mathrm{~cm} \times 100 \mathrm{~cm}$. Quanti cartelloni ti occorrono al minimo, se vuoi tappezzare la parete senza sovrapposizioni e buchi tra un cartellone e l'altro?

Questo problema potrebbe essere considerato un compito di realtà (oppure un compito autentico, se risponde a un effettivo bisogno della classe). I bambini potrebbero procedere in diversi modi: calcolare le dimensioni della parete dell'aula per poterne calcolare l'area e poi stabilire quanti cartoncini rettangolari della misura data sono contenuti in essa; potrebbero effettuare delle azioni concrete per vedere quanti cartoncini di quelle dimensioni si possono utilizzare; potrebbero anche rendersi conto che il risultato dell'algoritmo che emerge dal calcolo dell'area non è lo stesso di quello ottenuto quando si effettua una verifica operativa.

Il commerciante (problema standard)

II signor Giorgio fa il commerciante. Compra 436 penne a $€ 0,80$ ciascuna. Rivende la metà delle penne e incassa $€ 266,00$. Quanto guadagna per ogni penna?

Si tratta di un classico problema scolastico standard sulla compravendita: il testo è brevissimo e, tra i dati numerici, quello che potrebbe spiazzare qualche bambino è

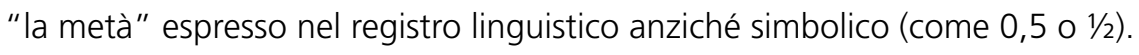

3. Un problema a righe è un problema caratterizzato da una profonda integrazione fra l'aspetto matematico e quello narrativo, in contrapposizione al problema a quadretti, in cui l'attenzione dell'autore è limitata alla struttura matematica. In un problema a righe la lettura selettiva del testo non è una strategia vincente, perché i dettagli della storia sono tutti funzionali alla comprensione del problema (Zan, 2016, p.190) 
Gli alunni di Anna (esercizio anticipato)

La maestra Anna, parlando dei suoi allievi, disse: «La metà dei miei allievi ama solo la matematica. La quarta parte ama solo le scienze; 1/7 ama solo I'arte e il disegno. Inoltre tre alunni amano tutte le materie». Quanti sono complessivamente gli alunni di Anna?

Il problema è stato modificato a partire dal classico problema Gli alunni di Pitagora 4 . Si tratta di un testo che può essere presentato agli allievi di quinta come un esercizio anticipato. A questo proposito, D'Amore, Fandiño Pinilla e Marazzani (2004, p. 74) distinguono un problema da un esercizio anticipato: I'esercizio anticipato è un esercizio standard, di uso e consumo molto routinario nella scuola, ad un certo punto dell'iter scolastico; solo che viene proposto come testo stimolo prima di quel momento. Gli alunni, attraverso strategie e rappresentazioni spontanee, meglio se in un setting di piccolo gruppo, potrebbero arrivare alla soluzione, così come è avvenuto in classi sperimentali. Tale problema può mettere in difficoltà gli stessi docenti che lo leggono per la prima volta oppure, ad una lettura poco attenta, potrebbe essere scambiato per un classico problema di calcolo di frazioni oppure, ancora, interpretato come problema a cui manca un dato.

II rettangolo (prova simil-Invalsi)

Marco dice: «Se raddoppia la misura del perimetro di un rettangolo, anche la sua area raddoppia».

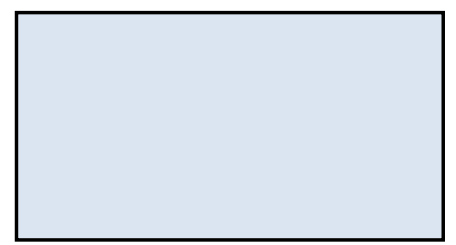

$\begin{array}{lll}\text { Sei d'accordo con lui? } & \text { Si }\end{array}$

Giustifica la tua risposta

Il problema è privo di dati numerici. Mette alla prova la presenza nei bambini della misconcezione secondo cui ci sia una relazione di proporzionalità tra misura del perimetro e misura dell'area, per la quale nel momento in cui si modifica una si deve modificare anche l'altra. I bambini possono verificare con disegni o altre strategie la falsa affermazione e sono chiamati esplicitamente a giustificare le conclusioni a cui sono arrivati. Di proposito non sono stati inseriti nel testo elementi che richiamano un contesto reale.

Il giardino di Torquato ${ }^{5}$ (non standard inclusivo)

Questo è il giardino del signor Torquato:

4. https://matematichiamoblog.wordpress.com/2016/03/26/quanti-sono-gli-alunni-di-pitagora/ 5. Il problema "II giardino di Torquato" fa parte di una raccolta di problemi tratti dal RMT (Rally matematico transalpino) 


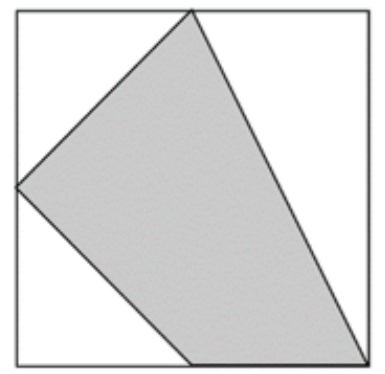

Nella parte grigia egli ha piantato fiori e ha seminato a prato la parte bianca. ॥ signor Torquato osserva il suo giardino e si chiede: "Sarà maggiore I'area della parte con i fiori o quella della parte con il prato?»

Si tratta ancora di un problema privo di dati numerici.

La figura che è all'interno del quadrato è un quadrilatero non standard. La risoluzione del problema, che mette in gioco i concetti di area e di equiscomponibilità, può prevedere la messa in gioco di diversi procedimenti risolutivi.

\section{L'analisi dei dati e l'interpretazione dei risultati}

\subsection{Le caratteristiche di un problema matematico nelle convinzioni dei docenti}

Partiamo da una prima domanda, posta ai 45 insegnanti.

1. Quali caratteristiche deve avere, secondo te, un problema matematico? II contenuto delle risposte date dai docenti alla domanda è stato analizzato con il software NVIVO 11, già menzionato. In prima istanza è stata effettuata una word frequency query che ha permesso di ricercare le parole più frequenti nelle risposte dei docenti. Tale interrogazione ha condotto alla produzione del grafico in Figura 1 e ai dati riassunti in Tabella 1.

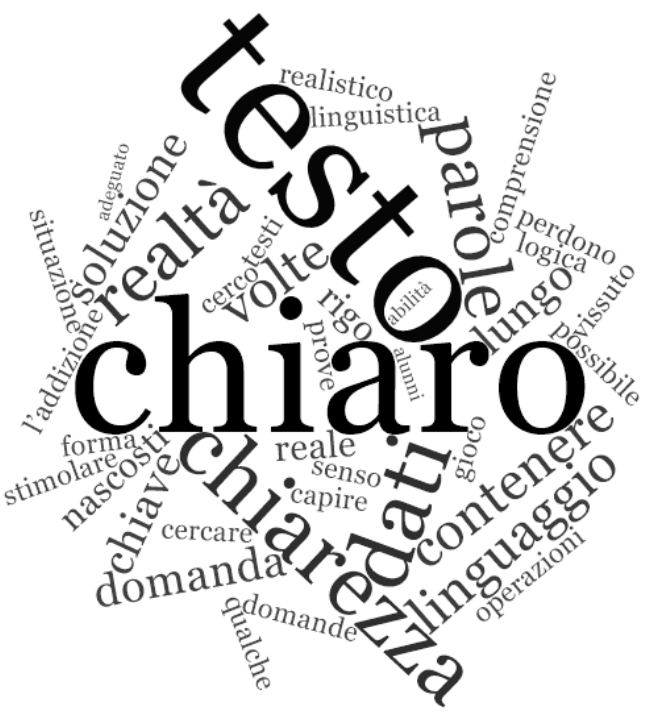


Tabella 1

Word frequency query

relativa alle caratteristiche

di un problema mate-

matico.

\begin{tabular}{|c|c|c|}
\hline Word & Lenght & Count \\
\hline chiaro & 6 & 21 \\
\hline testo & 5 & 20 \\
\hline chiarezza & 9 & 10 \\
\hline dati & 4 & 8 \\
\hline parole & 6 & 8 \\
\hline realtà & 6 & \\
\hline
\end{tabular}

Il grafico mostra che i termini chiaro, chiarezza, testo sono di gran lunga i più frequenti. Ma quale significato viene attribuito dagli insegnanti al termine chiaro? Cosa vuol dire che un problema deve essere chiaro? Per poter meglio interpretare questo aspetto, si è deciso di effettuare un'analisi più approfondita dei testi nei quali tali parole erano contenute e si è arrivati alla conclusione che i diversi significati attribuiti dai docenti sono riconducibili a una duplice classificazione:

1. chiaro in relazione a processi di comprensione linguistica;

2. chiaro in relazione a processi di comprensione matematica.

Per esemplificare, si riporta qualche testo tratto dalle risposte di alcuni docenti:

1. «Un problema deve essere chiaro, non molto lungo, non deve contenere più di due domande, più di due operazioni, perché poi i bambini si perdono. Deve contenere i dati, anche quelli nascosti, ovvio, i bambini poi ci arrivano, un po' discutendo, le classiche parole magiche, le parole chiave, che devono essere contenute nel testo, nella domanda, cerco di avviarli in forma di gioco».

2. «Deve essere chiaro, non tanto lungo. Partendo dalle domande esplicite, poi quelle implicite. Ho visto in alcuni testi che ci sono degli asterischi dove dovrebbe essere inserita la domanda implicita. È bene lasciarli e poi piano piano toglierli».

3. «Una caratteristica principale è la chiarezza. Non deve avere troppi dati, altrimenti i bambini vanno in tilt e non sono in grado di capire il testo del problema. Per chiarezza intendo il testo e anche la chiarezza dei dati matematici che non devono essere troppo arzigogolati».

4. «Deve essere non di esecuzione, di regole, ma di applicazione logica. Per i bambini che hanno difficoltà frasi brevi, a tappe; la chiarezza nella richiesta per tutti».

5. «Deve essere chiaro, deve essere un po' una sfida per chi lo fa. A volte sono poco chiari nella struttura del testo, nella comprensione del testo. A volte sono ingannevoli, perché sono messi in un italiano scorretto».

6. «Deve spingere al ragionamento. La componente logica è fondamentale. Poi la chiarezza del testo, soprattutto per bambini abbastanza piccoli. I termini devono essere comprensibili».

Le parole dei primi tre docenti (interventi 1, 2, e 3) mettono in evidenza che il costrutto "chiaro" è inteso in riferimento a un "testo non troppo lungo» e ciò potrebbe essere considerato un elemento attinente ai processi linguistici; lo stesso costrutto, però, è anche inteso, dal punto di vista dei processi matematici, in riferimento 
a un testo che "non deve avere più di due domande e più di due operazioni», corredato dalle parole chiave, con la presenza anche di stratagemmi (gli asterischi) che permettono ai bambini di individuare le domande nascoste. In questo caso i docenti sembrano essere più interessati al risultato conseguito dagli allievi che devono risolvere il problema (e cioè al prodotto finale rispetto al processo). Gli interventi 4, 5 , e 6 mettono in evidenza che il costrutto "chiaro" è inteso sicuramente rispetto ai processi linguistici: «italiano corretto, frasi brevi, a tappe, termini comprensibili». Dal punto di vista dei processi matematici si mette in evidenza che «deve essere un testo di applicazione logica, una sfida per chi lo fa, deve spingere al ragionamento». Tali affermazioni sembrano andare in una direzione di interesse per il processo risolutivo.

Nella Tabella 2 sono riportate due liste di espressioni linguistiche degli insegnanti, riferibili ai due significati individuati.

Tabella 2

Espressioni linguistiche dei docenti sul significato del termine "chiaro".

\begin{tabular}{|l|l|}
\hline \multicolumn{1}{|c|}{ Chiaro (processi linguistici) } & \multicolumn{1}{c|}{ Chiaro (processi matematici) } \\
\hline Messo in un italiano semplice & $\begin{array}{l}\text { Non deve contenere più di due domande e due } \\
\text { operazioni }\end{array}$ \\
\hline I termini devono essere comprensibili & $\begin{array}{l}\text { Deve contenere i dati, anche nascosti e le parole } \\
\text { chiave, nel testo e nella domanda }\end{array}$ \\
\hline Breve, conciso & $\begin{array}{l}\text { Non deve contenere troppi dati, altrimenti i bam- } \\
\text { bini vanno in tilt }\end{array}$ \\
\hline A volte sono messi in un italiano scorretto & $\begin{array}{l}\text { In alcuni testi ci sono gli asterischi, dove andreb- } \\
\text { be inserita la domanda implicita }\end{array}$ \\
\hline Evitare le subordinate & $\begin{array}{l}\text { Chiarezza dei dati matematici, che non devono } \\
\text { essere troppo arzigogolati }\end{array}$ \\
\hline Sintassi e forma del testo & A tappe \\
\hline Chiaro nell'esposizione, ben comprensibile & $\begin{array}{l}\text { Se immediatamente colgo i dati, il quesito, il re- } \\
\text { sto viene da sé }\end{array}$ \\
\hline Non deve essere troppo lungo & \\
\hline Testo spalmato e chiaro & \\
\hline Limpido e lineare & \\
\hline
\end{tabular}

\subsection{Le graduatorie dei problemi}

Sono state poste ai 45 docenti le seguenti domande:

- Hai letto e analizzato i 7 problemi. Ora componi una graduatoria, mettendo al primo posto il problema che ti piace di più e all'ultimo posto quello che ti piace di meno. Motiva le tue scelte.

- Componi, ora, una seconda graduatoria, mettendo al primo posto il problema che ritieni più adatto da proporre in classe ai tuoi futuri alunni di quinta e all'ultimo quello che ritieni meno adatto. Motiva le tue scelte.

Nel grafico presentato in Figura 2 sono rappresentate, nella colonna a sinistra, le percentuali di collocazione al primo o al secondo posto di ciascuno dei 7 problemi e, nella colonna a destra, le percentuali di collocazione al penultimo o all'ultimo posto degli stessi problemi. 


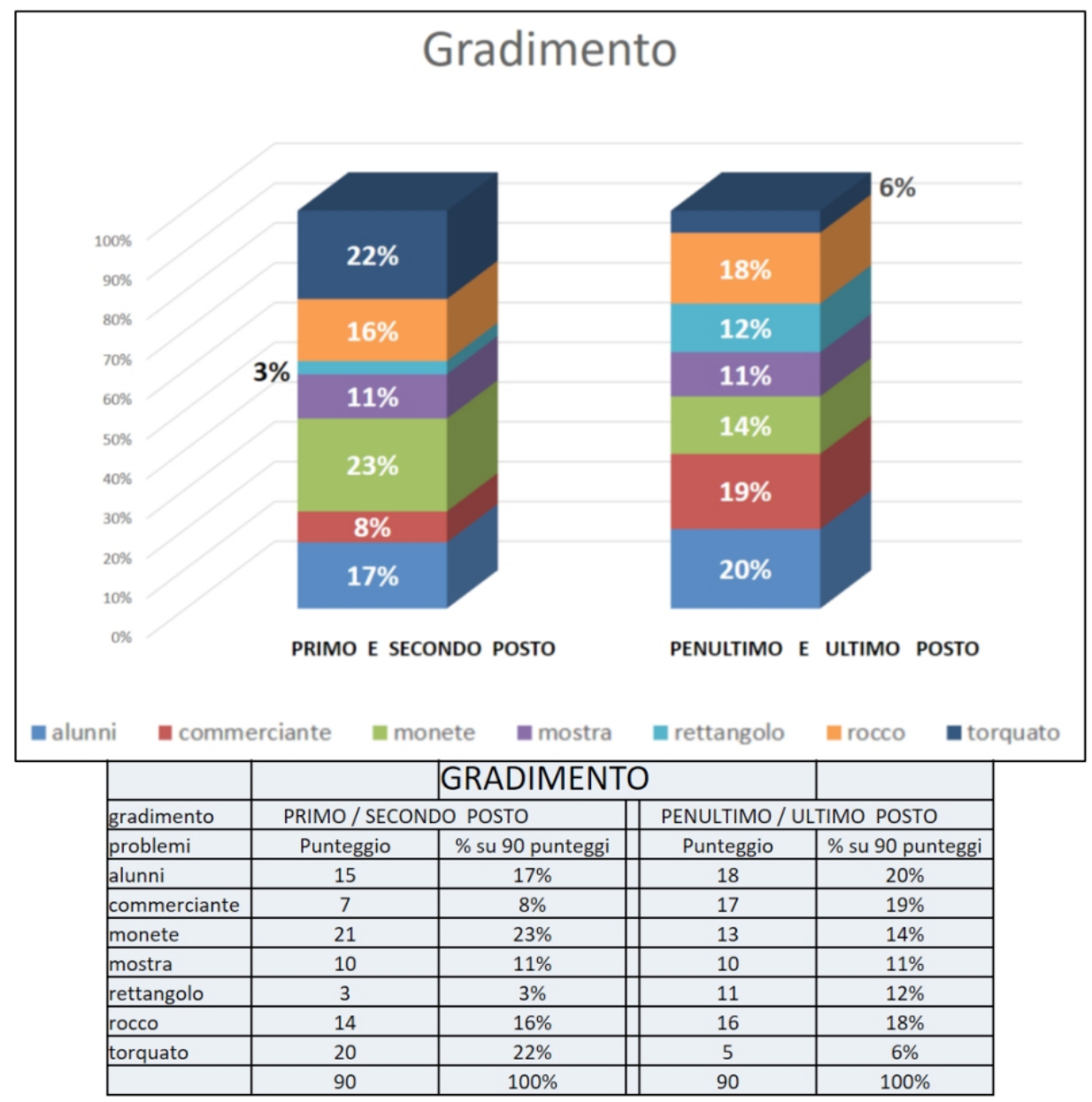

Il grafico mostra che un problema molto apprezzato dai docenti è stato il problema non standard inclusivo "Il giardino di Torquato", che è indicato per il $22 \%$ al primo $\mathrm{o}$ al secondo posto nella graduatoria di gradimento e solo per il $6 \%$ al penultimo o ultimo posto. Una lettura più analitica dei dati raccolti mostra che questo problema non è stato mai collocato dai docenti all'ultimo posto.

I problemi che mostrano un buon equilibrio nella collocazione al primo o al secondo posto da una parte e al penultimo o all'ultimo posto dall'altra, sono: "Rocco e il suo giardino" (16\% e 18\%), "La mostra in aula" (11\% e 11\%), "Gli alunni di Anna" (17\% e $20 \%)$.

II problema narrativo "Le monete" è indicato dal $23 \%$ degli insegnanti al primo o al secondo posto (la più alta percentuale); una buona percentuale di docenti, tuttavia, lo rifiuta e lo mette al penultimo o all'ultimo posto (14\%). II secondo problema standard "Il commerciante" è generalmente poco gradito: è per l' $8 \%$ dei docenti al primo o al secondo posto e per il $19 \%$ al penultimo o all'ultimo posto. Lo stesso vale per il problema "Il rettangolo", che è stato poco indicato nelle prime posizioni e più indicato nelle ultime posizioni.

In Figura 3 sono rappresentate le percentuali relative alla dichiarata applicabilità in aula dei problemi da parte dei docenti. 


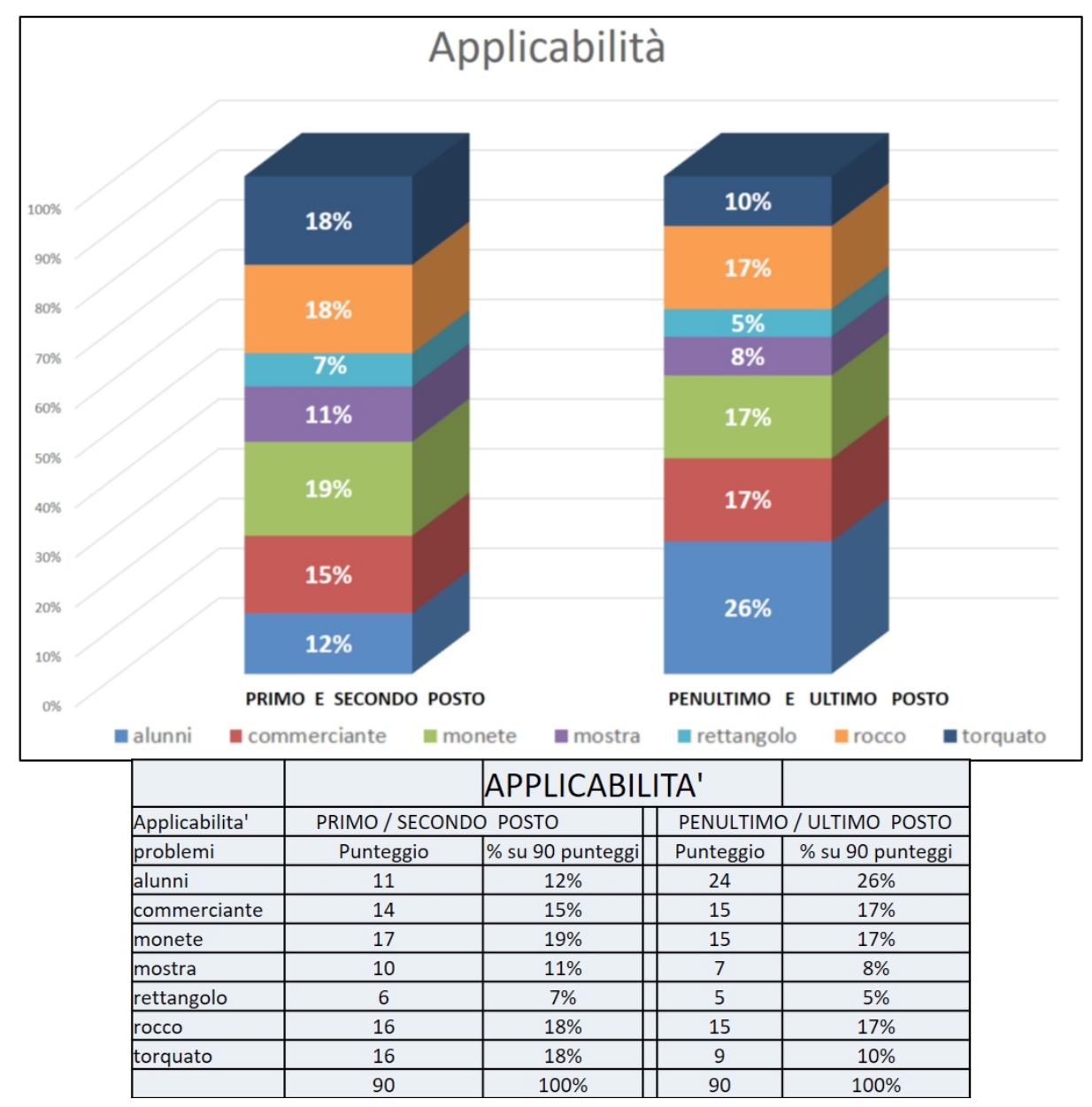

Dall'analisi del grafico, rileviamo che i due problemi "Il giardino di Torquato" e "Le monete" mantengono percentuali buone anche per l'applicabilità (18\% e 19\% al primo o al secondo posto) anche se "Le monete" è considerato meno applicabile rispetto a "Il giardino di Torquato" (17\% versus $10 \%$ al penultimo o all'ultimo posto).

L'esercizio anticipato "Gli alunni di Anna" è considerato poco applicabile (27\% al penultimo o ultimo posto). II problema standard "Il commerciante" ha una posizione equilibrata tra primi e ultimi posti (15\% e 17\%), così anche "Rocco e il suo giardino" (18\% e 17\%) e "Una mostra in aula" (11\% e $8 \%)$. I problema "I rettangolo" anche in questa graduatoria ottiene una percentuale bassa per l'applicabilità (7\%), anche per la non applicabilità $(6 \%)$, considerando che in caso di non applicabilità il problema ha occupato raramente le posizioni più base della classifica. Naturalmente, trattandosi di un campione poco numeroso, possiamo parlare di tendenze, che andranno poi confermate in futuri studi effettuati su campioni estesi. Si confrontano, ora, le due graduatorie, di gradimento e di applicabilità, per verificarne i gradi di corrispondenza (Figura 4). 
Figura 4

Confronto tra i grafici rappresentativi delle due graduatorie: gradimento e applicabilità.

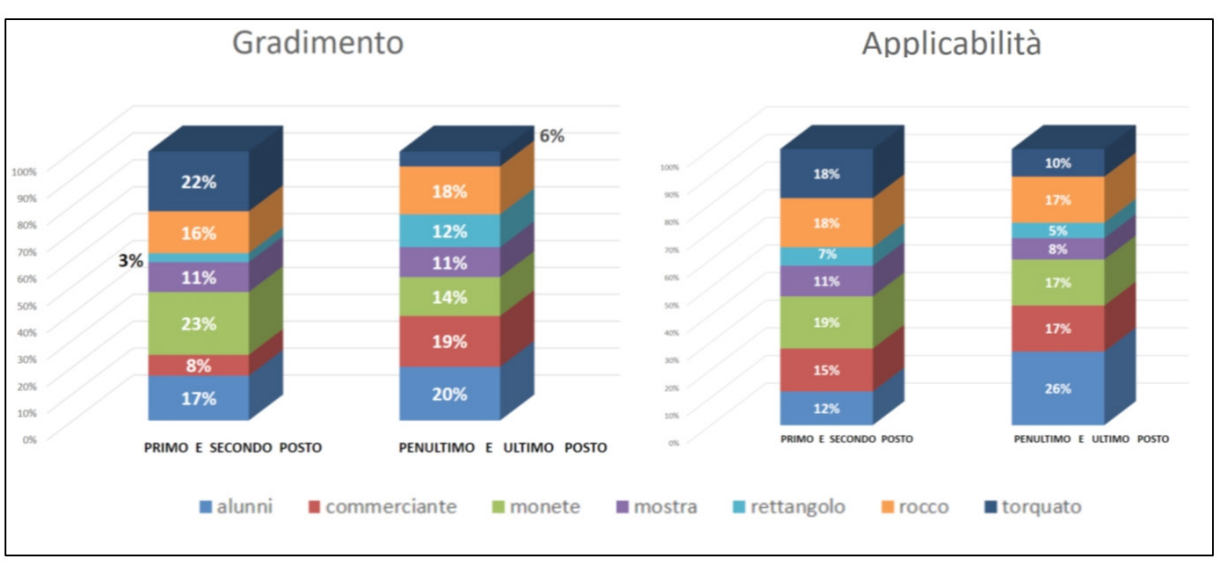

Alcune osservazioni a riguardo. II problema narrativo "Le monete" è generalmente gradito ai docenti, ma una parte di loro ritiene di non poterlo rendere applicabile in aula. II problema non standard inclusivo "II giardino di Torquato" è gradito e ritenuto più applicabile del precedente. "Il commerciante" (problema standard-aritmetico) è poco gradito da un buon numero di insegnanti, che comunque lo scelgono rispetto alla sua applicabilità in aula.

L'esercizio anticipato "Gli alunni di Anna", pur essendo un problema gradito, perde in applicabilità.

"Una mostra in aula" (compito di realtà) e "Il rettangolo" (prova simil-Invalsi) sono meno presenti nelle posizioni estreme al positivo o al negativo (I e II posto oppure VI e VII). Ciò significa che tali problemi occupano spesso le posizioni intermedie delle classifiche; gli insegnanti non si mostrano favorevoli verso di essi, ma nemmeno li rifiutano. In particolare, il problema scelto come compito di realtà, "Una mostra in aula", ha messo in luce alcune perplessità da parte degli intervistati, i quali spesso ne hanno commentato l'applicabilità: «è difficile per i bambini misurare la parete»; "anche se ambientato in un contesto reale non è autentico e non necessariamente coinvolgerebbe gli allievi». Più volte i docenti hanno proposto altre versioni. Si riporta, a titolo d'esempio, la proposta effettuata da un'insegnante:

«lo inizierei a porre semplicemente il problema. "Dobbiamo fare questa cosa. Sentiamo che consigli mi date per sistemare questa cosa". Poi sentiamo un po' cosa viene fuori e poi a imbuto ci andiamo a infilare in conversazioni più centrate sulle questioni matematiche. In prima istanza: "Noi abbiamo questo mucchio di disegni. Se volessimo sistemarli, in che modo potremmo fare?". Ogni gruppetto espone la sua proposta. L'importante è lavorare in un contesto protetto, in un contesto guidato e sereno, dove nessuno si sente giudicato e le risposte dell'insegnante non hanno un significato di giudizio».

Focalizzando l'attenzione sul rapporto tra problemi proposti e convinzioni dei docenti, si può dire che in particolare alcuni problemi sembrano aver stimolato maggiormente le reazioni dei docenti.

A questo punto dell'analisi, sorgono nuovi interrogativi:

1. Quali sono le motivazioni addotte dai docenti per spiegare il gradimento dei testi "Il giardino di Torquato" e "Le monete"? 
2. Quali sono le motivazioni addotte dai docenti per spiegare il non gradimento dei due problemi "Il giardino di Torquato" e "Le monete"?

3. Quali sono le motivazioni che i docenti hanno dato per spiegare lo scarso gradimento del testo "Il commerciante"?

4. Perché i docenti hanno considerato il problema "II commerciante" applicabile in aula?

5. Perché il testo "Il giardino di Torquato" è stato gradito e è stato anche considerato più applicabile in classe, rispetto a "Le monete"?

\subsection{Primo piano su alcuni problemi}

Emerge in modo evidente che gli insegnanti intervistati hanno convinzioni diverse rispetto ai sette problemi presentati e in particolare emerge una maggiore variabilità rispetto ai tre problemi "Il giardino di Torquato", "Le monete" e "Il commerciante", che hanno sollecitato pareri contrastanti e, alcune volte, anche una bella partecipazione dei docenti intervistati, sia dal punto di vista della valutazione cognitiva che per la reazione emotiva.

Il problema "Il giardino di Torquato" è stato collocato al primo o al secondo posto nella graduatoria di gradimento dal $22 \%$ degli insegnanti per i seguenti motivi.

«È diverso dal solito, non è il classico problema geometrico; è un problema senza numeri e presenta una figura irregolare».

«Richiede ragionamento».

«Tutti potrebbero arrivare alla soluzione, perché si possono usare strategie diverse, anche il ritaglio; è accessibile anche ai bambini che non ragionano». «Stimola il pensiero divergente, nel senso che devi far vedere le figure».

«È uno strumento per costruire competenze».

«Stimola la discussione e la creatività dei bambini».

«Sviluppa la capacità di vedere in geometria».

«Permette di riuscire a vedere, immaginarsi movimenti e spostamenti che portano a valutare equiestensione».

Il problema "Il giardino di Torquato", di contro, è stato collocato al penultimo e ultimo posto nella graduatoria di gradimento nel $6 \%$ dei casi per i seguenti motivi:

«Non ci sono dati esplicitati».

«È diverso dagli altri».

«La figura potrebbe metterli in difficoltà».

«Lo vorrei risolvere prima io».

«È un lavoro a livello di figure geometriche, molto complesso».

«È un problema che può essere risolto solo dai bambini intuitivi».

«Devono ragionarci, applicare le regole della matematica, ma con il ragionamento».

Il problema "Le monete" è stato collocato al primo o al secondo posto nella graduatoria di gradimento dal $23 \%$ dei docenti; si riportano alcune delle motivazioni addotte:

«È logico, coinvolge la logica e i procedimenti logici». 
«Stimola la discussione».

«Stimola il ragionamento».

«Permette diverse interpretazioni (socio-affettiva e matematica)».

«È uno strumento per costruire competenze».

«È interessante».

Di contro, lo stesso problema è stato collocato al penultimo e ultimo posto nella graduatoria di gradimento dal $14 \%$ degli insegnanti per i seguenti motivi.

«È complicatissimo dal punto di vista del linguaggio».

«[Gli allievi] cercherebbero subito i numeri».

«Devono ragionare tantissimo».

«Lo proporrei non come testo; lo farei rappresentare, lo racconterei».

«È farraginoso, ingarbugliato».

«Ha troppi dati numerici».

«Sui sussidiari non troverai mai un problema così».

«ll bambino si può spaventare; lo dividerei in pezzetti; analizzando i pezzetti sarebbe una bella sfida...».

II problema "Il commerciante" è stato collocato al primo o al secondo posto dall' $8 \%$ degli insegnanti nella graduatoria di gradimento; riportiamo una delle motivazioni addotte e argomentate:

«Questo è proprio sintetico al massimo. Questi sono i classici problemi che si propongono. Sì, lo proporrei, sono quei problemi classici. E qui la discussione è molto più ampia perché loro non capiscono che un commerciante deve comprare anche lui, ci deve guadagnare perché ci deve guadagnare. C'è tutta prima una discussione sul perché c'è la spesa, perché c'è il ricavo, perché c'è il guadagno. È vero che c'è la formuletta, ma in realtà loro non lo vivono, perché non sono commercianti».

Lo stesso problema è stato collocato al penultimo o ultimo posto per il 19\% nella graduatoria di gradimento. Riportiamo una delle motivazioni addotte:

«Sono problemi di compravendita e sono su tutti i sussidiari. C'è un po' di argomentazione ma non è un'argomentazione. Una volta che è stato risolto da qualcuno, questo qualcuno non fa fatica a spiegare; è una soluzione abbastanza lineare è [...]. lo l'argomentazione la vedo come qualcosa di più creativo, qui di creativo non vedo quasi niente. Non è un problema che ti tocca più di tanto quando discuto in classe di queste robe qua [...]».

\subsection{Gli aspetti significativi dei tre problemi}

Si elencano, di seguito, gli aspetti che sono apparsi più significativi nell'analisi di questi tre problemi. Si riportano come esempi, a supporto di queste affermazioni, alcuni stralci delle interviste dei docenti.

1. Le stesse caratteristiche di uno specifico problema possono essere considerate elementi positivi per alcuni docenti ed elementi negativi per altri. 
Ad esempio, sul problema "Il giardino di Torquato" sono state fatte le seguenti considerazioni contrastanti:

"L'impatto è visivo e non ci sono dati esplicitati. No, non mi convince, perché diciamo che mancano dei dati ed è anche un lavoro a livello di figure geometriche abbastanza complesso».

«A me piace tantissimo questo. Lo proporrei sicuramente perché è un problema che sviluppa proprio la capacità di vedere in geometria. Potrebbero affrontarlo come lavoro individuale: ciascuno vede a livello visivo, poi discussione con tutta la classe; poi farei un lavoro per gruppi eterogenei per risposte».

2. Uno stesso problema può essere considerato facile o difficile in riferimento ad uno stesso target di alunni.

Ad esempio, a questo proposito, sul problema "Il commerciante" è stato detto:

«Non ci capiscono quasi niente. Per loro rivendere è guadagnare. Ecco per esempio la discussione, che però non è una discussione, non è un'argomentazione ma una confusione sui termini, sui significati. Rivende la metà delle penne e incassa. Quanto guadagna per ogni penna, per loro è la stessa cosa. La differenza non esiste».

«Non so se lo proporrei. Mi sembra un problema molto difficile, questo [...]». "C'è tutta prima una discussione sul perché c'è la spesa perché c'è il ricavo, perché c'è il guadagno. È vero che c'è la formuletta, ma in realtà loro non lo vivono, perché non sono commercianti».

3. Non è tanto importante il problema in sé quanto le modalità in cui l'insegnante presenta un problema, a renderlo un buon problema o un problema di poco valore. Sempre sul problema "Il commerciante" alcuni insegnanti affermano:

«Per me dipende da come lo poni. La discussione nasce perchéil commerciante... Che cosa fa? Perché la metà? Poi il prezzo unitario, il prezzo totale. C'è di tutto e di più. Ė molto significativo. È anche sempre un problema di esperienza e vita reale. Noi poi in questi giorni stiamo proprio parlando del commerciante: chi è, che fa. I bambini, devo dire, sono sempre stimolati».

"Qui c'è il discorso dell'ingrosso che secondo me è un concetto che si può introdurre. II commerciante compra dove vendono gli stock, addirittura potrebbe andare alla fabbrica della BIC. Stimola tanto l'argomentazione e la discussione per questa cosa che dicevo di come funziona il commercio, che nemmeno io lo so».

4. Ci sono alcuni problemi che più di altri sono considerati inclusivi, in quanto danno la possibilità agli allievi di utilizzare vari tipi di strategie didattiche.

Per il problema "Il giardino di Torquato" ad esempio, alcuni docenti affermano: «Un problema che non ha numeri la sollecita la discussione, perché tu devi trovare una strategia; a me è venuta quella della sovrapposizione, ma magari a un bambino viene in mente un'altra strategia, che è diversa da quella aritmetica, mi sembra abbastanza semplice; dovrebbe essere accessibile anche 
al bambino che ragiona di meno».

«Hanno fatto ipotesi anche quelli che non ci arrivano; hanno detto di tutto e di più. Noi accettiamo tutto. Questo è lo scopo: di stimolarli comunque a discutere e ad intervenire. In questo i nostri sono abbastanza sicuri. Facciamo sempre questa pedagogia del non timore e viene fuori di tutto e di più».

"Stimola un po' il pensiero divergente, nel senso che devi vedere le figure e far fare una cosa su cui punto tantissimo: vedere la geometria non solo come figurine, ma scomporre e ricomporre le figure. Perché ognuno deve mettersi in discussione con sé stesso».

5. I problemi che particolarmente stimolano discussione e argomentazione possono essere proposti solo ad alcuni bambini, perché si pensa che la maggior parte degli alunni della propria classe non riesca mai ad arrivare alla soluzione. Per esempio, sul problema "Le monete" si riporta il pensiero di una docente:

«Questo è un problema che io proporrei, però non a tutta la classe. Perché io ho la presenza di un portatore di handicap, la presenza di due DSA gravi e ci sono anche bambini che non hanno queste attenzioni per il ragionamento. lo lo proporrei a un gruppo della classe omogeneo. Questo lo vedo adatto a bambini che riescono a trovare quelle strategie diverse».

\subsection{Problemi e libri di testo}

Si affronta infine un'ulteriore questione delicata e didatticamente importante: i problemi presenti nei libri di testo. Spesso accade che, pur dichiarando il rispetto delle Indicazioni Nazionali per il curricolo, editori e autori seguano di fatto altre strade, più orientate a riprodurre una didattica di tipo tradizionale. Considerando anche che molti docenti danno grande importanza ai libri di testo, anche per ragioni pratiche e di condivisione con i colleghi, appare di particolare rilevanza investigare sulle convinzioni che i docenti hanno in proposito. È stato chiesto ai docenti, nel corso dell'intervista:

- Sei soddisfatto/a dei problemi proposti sui libri di testo? Motiva la tua risposta.

- Quali suggerimenti daresti ai responsabili di una casa editrice per migliorare i problemi proposti sui libri di testo?

Le risposte dei docenti alla prima delle due domande sono state classificate a posteriori, individuando alcuni "punti di vista" sui problemi suggeriti dai contenuti delle risposte stesse:

- il punto di vista del docente esperto in matematica;

- il punto di vista del docente che riflette sull'utilità delle proposte per il proprio lavoro;

- il punto di vista dell'insegnante che "si mette nei panni" degli alunni;

- il punto di vista del docente professionista, che valuta l'aderenza dei problemi presenti sui libri di testo a problemi "ideali" che abbiano le caratteristiche auspicate nei documenti istituzionali, in particolare nel testo delle Indicazioni Nazionali per il curricolo.

Gli insegnanti intervistati si sono dichiarati alquanto insoddisfatti dei problemi presenti sui libri di testo e le motivazioni addotte sono state classificate sulla base dei punti di vista sopra menzionati, come illustrato in Tabella 3. 
Tabella 3

Come gli insegnanti motivano la mancata soddisfazione dei problemi proposti sui libri di testo.

\begin{tabular}{|c|c|c|c|}
\hline $\begin{array}{l}\text { Dal punto di vista } \\
\text { del matematico }\end{array}$ & $\begin{array}{l}\text { Dal punto di vista } \\
\text { dell'utilità pratica }\end{array}$ & $\begin{array}{l}\text { "Nei panni" } \\
\text { degli allievi }\end{array}$ & $\begin{array}{l}\text { Aderenza ai docu- } \\
\text { menti istituzionali }\end{array}$ \\
\hline $\begin{array}{l}\text { Stimolano poco } \\
\text { la logica }\end{array}$ & Semplificano il lavoro & $\begin{array}{l}\text { Linguaggio non } \\
\text { vicino ai bambini }\end{array}$ & $\begin{array}{l}\text { Non in linea con le } \\
\text { Indicazioni Nazionali }\end{array}$ \\
\hline $\begin{array}{l}\text { Sono esercizi e } \\
\text { problemi standard }\end{array}$ & Sono pochi & Banali & $\begin{array}{l}\text { Non utili per la } \\
\text { certificazione delle } \\
\text { competenze }\end{array}$ \\
\hline \multirow[t]{3}{*}{$\begin{array}{l}\text { Sono classificati per } \\
\text { argomento: moltipli- } \\
\text { cazioni, divisioni ecc. }\end{array}$} & $\begin{array}{l}\text { Si devono integrare } \\
\text { con altri reperiti da } \\
\text { siti o altri materiali } \\
\text { cartacei }\end{array}$ & Noiosi e ripetitivi & $\begin{array}{l}\text { Non stimolano il } \\
\text { ragionamento }\end{array}$ \\
\hline & $\begin{array}{l}\text { Devono essere } \\
\text { modificati }\end{array}$ & Testi poco chiari & $\begin{array}{l}\text { Non ci sono giochi } \\
\text { matematici né test } \\
\text { Invalsi }\end{array}$ \\
\hline & $\begin{array}{l}\text { Ogni insegnante si } \\
\text { regola a modo suo }\end{array}$ & $\begin{array}{l}\text { Troppo semplici o } \\
\text { troppo complicati }\end{array}$ & \\
\hline
\end{tabular}

Gli insegnanti intervistati hanno espresso i loro suggerimenti agli editori, così come è illustrato in Tabella 4.

\begin{tabular}{|c|c|c|c|}
\hline $\begin{array}{l}\text { Dal punto di vista } \\
\text { del matematico }\end{array}$ & $\begin{array}{l}\text { Dal punto di vista } \\
\text { dell'utilità pratica }\end{array}$ & $\begin{array}{l}\text { "Nei panni" } \\
\text { degli allievi }\end{array}$ & $\begin{array}{l}\text { Aderenza ai docu- } \\
\text { menti istituzionali }\end{array}$ \\
\hline $\begin{array}{l}\text { Inserire problemi aper- } \\
\text { ti e senza soluzione }\end{array}$ & $\begin{array}{l}\text { Inserire pochi problemi } \\
\text { ma buoni }\end{array}$ & $\begin{array}{l}\text { Spazio alla creatività } \\
\text { e a modelli intuitivi } \\
\text { diversi }\end{array}$ & $\begin{array}{l}\text { Inserire problemi più } \\
\text { autentici e legati al } \\
\text { reale, vivibili, concreti, } \\
\text { legati ai tempi, al con- } \\
\text { testo dei bambini }\end{array}$ \\
\hline $\begin{array}{l}\text { Inserire } \\
\text { problemi storici }\end{array}$ & $\begin{array}{l}\text { Affiancare guide per i } \\
\text { docenti dove si danno } \\
\text { proposte e anche } \\
\text { soluzioni }\end{array}$ & $\begin{array}{l}\text { Inserire problemi gio- } \\
\text { cosi, giocati, creativi }\end{array}$ & $\begin{array}{l}\text { Non dare delle regole } \\
\text { di risoluzione ai bam- } \\
\text { bini, per permettere } \\
\text { di lavorarci con il loro } \\
\text { ragionamento }\end{array}$ \\
\hline $\begin{array}{l}\text { Differenziare i } \\
\text { problemi sullo stesso } \\
\text { argomento }\end{array}$ & $\begin{array}{l}\text { Gli autori sono gli } \\
\text { esperti, quindi non si } \\
\text { mette in discussione } \\
\text { I'utilità dei problemi } \\
\text { proposti }\end{array}$ & $\begin{array}{l}\text { Far fare ai bambini } \\
\text { cose in pratica }\end{array}$ & $\begin{array}{l}\text { Inserire esercizi ma } \\
\text { anche problemi che } \\
\text { stimolino il ragiona- } \\
\text { mento }\end{array}$ \\
\hline \multirow[t]{2}{*}{$\begin{array}{l}\text { Inserire problemi di } \\
\text { vari livelli e gradi di } \\
\text { difficoltà }\end{array}$} & Arricchire i testi & $\begin{array}{l}\text { Inserire problemi legati } \\
\text { a vocaboli semplici, } \\
\text { per ceti bassi }\end{array}$ & $\begin{array}{l}\text { Inserire problemi } \\
\text { stimolanti, che diano } \\
\text { modo di discutere }\end{array}$ \\
\hline & $\begin{array}{l}\text { Dare agli insegnanti } \\
\text { degli spunti per } \\
\text { costruire problemi ad } \\
\text { hoc, in situazione }\end{array}$ & $\begin{array}{l}\text { Dare la possibilità di } \\
\text { uno o più step: un } \\
\text { bambino arriva ad un } \\
\text { punto, un altro bam- } \\
\text { bino ad un altro }\end{array}$ & $\begin{array}{l}\text { Proporre prove } \\
\text { simil-Invalsi }\end{array}$ \\
\hline
\end{tabular}

Analizzando le tabelle e tenendo conto dei dati acquisiti, grazie a un'analisi ulteriormente approfondita dei testi delle risposte, si possono effettuare le seguenti considerazioni: 
1. I problemi dei libri di testo non soddisfano molto i docenti intervistati per quanto riguarda aspetti che sono riferibili a questioni più prettamente matematiche; sono pochi i "veri" problemi presenti, non rispondono ai bisogni degli allievi e non sono in linea con i documenti istituzionali, nello specifico con le Indicazioni Nazionali per il curricolo. È chiaro che i diversi aspetti citati sono in relazione tra loro: ad esempio, laddove si chiede di inserire problemi che siano "giocosi, giocati, creativi" ci si riferisce ai bisogni dei bambini, ma anche ad aspetti matematici e all'aderenza alle Indicazioni Nazionali.

2. Gli insegnanti mostrano di essere abbastanza consapevoli delle differenze che ci sono tra esercizi e problemi, ma sono pochi coloro che mostrano di conoscere la letteratura di riferimento e di averne fatto oggetto di riflessione professionale, individualmente o a scuola. Molti insegnanti lamentano la presenza massiccia, nei libri di testo, di esercizi utili per verificare l'acquisizione di regole apprese in aula, piuttosto che di problemi, che possono mettere in gioco: ragionamento, vari modelli intuitivi, creatività degli allievi.

3. Per quanto riguarda le caratteristiche specifiche che i problemi dovrebbero avere, si insiste molto su quanto i problemi disponibili siano noiosi, ripetitivi, a volte banali, espressi in un linguaggio poco vicino ai bambini e poco chiaro. Per questi ultimi aspetti i problemi proposti dai libri di testo non sono ritenuti in linea con il testo delle Indicazioni Nazionali.

4. Per quel che riguarda gli aspetti più prettamente pratici per il lavoro in aula, gli insegnanti intervistati si ritengono soddisfatti, perché hanno la possibilità di avere dei materiali da utilizzare, anche se spesso devono integrarli con altri problemi oppure sono costretti a modificare i testi proposti, per renderli più chiari o più significativi. Alcuni insegnanti credono che siano proprio gli autori dei libri i veri esperti e dunque non se la sentono di proporre alcun cambiamento, riponendo in loro (gli autori) assoluta fiducia.

5. Gli insegnanti intervistati si mostrano molto propositivi e creativi, nel momento in cui sono invitati a dare consigli ai responsabili di una casa editrice per poter migliorare le proposte sui libri scolastici e parascolastici; essi utilizzano, nel definire i problemi "desiderati", tanti aggettivi che fanno riferimento a concetti importanti presenti nelle Indicazioni Nazionali: autentici, legati al reale, vivibili, concreti, legati ai tempi, legati al contesto dei bambini, giocosi, giocati, creativi. Tali aggettivi evidenziano le aspettative e i bisogni dei docenti di avere a disposizione problemi "diversi" per i loro allievi. Gli insegnanti insistono anche sulla necessità che si curi maggiormente la chiarezza dei testi, anche per favorire l'accessibilità ai problemi da parte dei bambini di ceto basso, cercando sempre però di non cadere nella banalità.

6. Alcuni insegnanti manifestano il bisogno di avere a disposizione problemi che rassicurino gli alunni (e forse loro stessi): problemi a più step, per esempio, oppure graduati, che prevedano diversi gradi di soluzioni, dal più facile al più difficile. Tali problemi darebbero ai bambini la possibilità di essere gratificati dal raggiungimento di un risultato, anche se solo ai primi livelli di difficoltà. Tali problemi darebbero anche modo ai docenti di monitorare i livelli di competenza raggiunti dai loro allievi. 


\section{Conclusioni: discussione dei risultati e implicazioni didattiche}

Nella prima parte dell'intervista gli insegnanti hanno analizzato sette problemi, selezionati e modificati appositamente, assumendo di essere confrontati con sei diverse tipologie: problema standard, problema narrativo, compito di realtà, esercizio anticipato, quesito simil-Invalsi, problema non standard inclusivo. Ciascuno dei sette problemi ha determinato, da parte degli insegnanti intervistati, risposte ben connotate, che sono state acquisite, analizzate e interpretate. I docenti si sono espressi in modo diametralmente opposto nei confronti di uno stesso problema, e ciò è apparso in modo evidente nel corso dell'analisi e della valutazione del problema narrativo "Le monete". Grazie alle due diverse "classifiche", di gradimento e di applicabilità in aula, è stato possibile prendere atto di eventuali incongruenze tra ciò che gli insegnanti dichiarano di apprezzare e ciò che invece sentono di poter proporre in aula, lavorando con i propri allievi.

Alcuni docenti hanno modificato le loro convinzioni su alcuni problemi nel corso dell'intervista stessa; si può ipotizzare che ci sia stato un inizio di cambio di convinzioni, proprio grazie alla possibilità di avere un "tempo dedicato" per poter riflettere su una vasta gamma di prove, per conoscerle meglio, valorizzandone le specifiche caratteristiche e potenzialità. Ciò ha "smosso" in alcuni docenti il proposito di poter modificare le proprie scelte didattiche e di portare la classe all'altezza di tali compiti, anziché la decisione di non somministrarli.

Una classificazione dei problemi matematici, così come è stata proposta nel corso delle interviste effettuate, potrebbe costituire uno spunto da cui partire per attuare confronti "in orizzontale", tra i docenti delle diverse classi, ma anche una riflessione da effettuare "in verticale", nei contesti di costruzione di curricula che abbracciano tutto il ciclo scolastico, dalla scuola dell'infanzia alla scuola media.

In questo modo i docenti potrebbero approfondire le potenzialità formative dei diversi problemi, così come potrebbero individuarne i limiti. Ciò avrebbe il beneficio di sviluppare un atteggiamento critico e costruttivo rispetto alla grande quantità di problemi e di prove di diversa natura che i docenti ritrovano nei testi scolastici e parascolastici, per poter operare una scelta oculata, consapevole e mirata dei problemi da presentare agli alunni, ipotizzando, perché no, una revisione e una riscrittura degli stessi, anche eventualmente in collaborazione con gli alunni.

Una molteplicità di proposte da parte degli insegnanti potrebbe inoltre valorizzare più stili cognitivi; sollecitare molteplici strategie rappresentative e comunicative; sviluppare e consolidare importanti competenze matematiche e non matematiche, in linea con i documenti nazionali e internazionali. Si ritiene che i traguardi formativi, auspicati per il problem solving sia nei documenti nazionali che nei documenti internazionali, siano raggiungibili puntando sulla molteplicità e sulla varietà delle proposte, e dunque presentando problemi di varia natura e di livello diverso, in modo tale da attivare le diverse intelligenze e gli svariati approcci personali degli alunni presenti nelle classi.

Si potrebbero poi configurare piste di autoformazione che abbiano anche il vantaggio di far acquisire agli insegnanti non solo una consapevolezza circa la propria visione della matematica e dei problemi matematici, ma anche, attraverso il confronto con i colleghi, delle possibili molteplicità di queste visioni (Zan, 2010). 


\section{Bibliografia}

Abelson, R. (1979) Differences between belief systems and knowledge systems. Cognitive Science, 3, 355-366.

Andrews, P. (2007). The curricular importance of mathematics. A comparison of English and Hungarian teachers' espoused beliefs. Journal of Curriculum Studies, 39(3), 317-338.

Andrews, P., \& Xenofontos, C. (2014). Analysing the relationship between the problem-solving-related beliefs, competence and teaching of three Cypriot primary teachers. Journal of Mathematics Teacher Education, 18(4), 299-325.

Baccaglini Frank, A., Di Martino, P., Natalini, R., \& Rosolini, G. (2018). Didattica della matematica. Mondadori, Milano.

Cai, J., \& Nie, B. (2007). Problem solving in Chinese mathematics education: Research and practice. ZDM, 39(6), 459-473.

Carey, S. (1999). Sources of conceptual change. In E. Scholnick, K. Nelson, S. Gelman \& P. Miller (Eds.), Conceptual Development (pp. 292-326). London: Lawrence Erlbaum.

Cohen, D. (1990). A revolution in one classroom: The case of Mrs. Oublier. Educational Evaluation and Policy Analysis, 12(3), 311-329.

D'Amore, B. (2014). I/ problema di matematica nella pratica didattica. Modena: Digital Docet.

D’Amore, B., \& Fandiño Pinilla, M. I. (2004). Cambi di convinzioni in insegnanti di matematica di scuola secondaria superiore in formazione iniziale. La matematica e la sua didattica, 3 , 27-50.

D'Amore, B., \& Fandiño Pinilla, M. I. (2006). Che problema i problemi! L'insegnamento della matematica e delle scienze integrate, 6, vol. 29 A-B, 645-664.

D’Amore, B., \& Fandiño Pinilla, M. I. (2009). La formazione degli insegnanti di matematica, problema pedagogico, didattico e culturale. In F. Frabboni \& M. L. Giovannini (Eds.), Professione insegnante (pp. 145-153). Milano: Franco Angeli.

D’Amore, B., Fandiño Pinilla, M. I., \& Marazzani, I. (2004). "Esercizi anticipati" e "zona di sviluppo prossimale": comportamento strategico e linguaggio comunicativo in attività di problem solving. La matematica e la sua didattica, 2, 71-95.

De Corte, E. (1995). Fostering cognitive growth: A perspective from research on mathematics learning and instruction. Educational Psychologist, 30(1), 37-46.

De Corte, E. (2004). Mainstreams and perspectives in research on learning (mathematics) from instruction. Applied Psychology: An International Review, 53(2), 279-310.

De Corte, E., Verschaffel, L., \& Op't Eynde, P. (2000). Self-regulation: A characteristic and a goal of mathematics learning. In M. Boekaerts, P. Pintrich \& M. Zeidner (Eds.), Handbook of Self-regulation (pp. 687-726). San Diego: Academic Press.

Di Martino, P. (2004). Difficoltà in matematica e sistemi di convinzioni. Tesi di dottorato. Università degli studi di Pisa.

Di Martino, P., \& Zan, R. (2017). Insegnare e apprendere la matematica con le Indicazioni nazionali. Ebook. Firenze: Giunti Scuola. 
Fandiño Pinilla, M. I. (2008). Molteplici aspetti dell'apprendimento della matematica. Valutare e intervenire in modo mirato e specifico. Trento: Erickson.

Furinghetti, F., \& Pehkonen, E. (2002). Rethinking Characterizations of Beliefs. In G.C. Leder, E. Pehkonen \& G. Törner (Eds.), Beliefs: A hidden Variable in Mathematics Education (pp. 39-57). Dordrecht, The Netherlands: Kluwer.

Green, T. F. (1971). The activities of teaching. New York: McGraw-Hill.

Lumbelli, L. (2006). Costruzione dell'ipotesi ed astrazione nella pedagogia sperimentale, 25-60.

McLeod, D. (1985). Affective issues in research on teaching mathematical problem solving. In E. Silver (Ed.), Teaching and Learning Mathematical Problem Solving: Multiple Research Perspectives. Hillsdale: Lawrence Erlbaum Associates Publishers.

McLeod, D. (1989). The role of affect in mathematical problem solving. In D. McLeod \& V. Adams (Eds.), Affect and mathematical problem solving: a new perspective. New York: Springer-Verlag.

McLeod, D. (1992): Research on affect in mathematics education: a reconceptualization. In D. Grouws (Ed.), Handbook of Research on Mathematics Learning and Teaching. New York: MacMillan.

MIUR. (2012). Indicazioni Nazionali per il curricolo della scuola dell'infanzia e del primo ciclo d'istruzione. Disponibile in http://www.indicazioninazionali.it/wp-content/uploads/2018/ 08/Indicazioni Annali Definitivo.pdf (consultato il 07.10.2019)

NCTM. (2010). Why is teaching with problem solving important to student learning? Disponibile in https://www.nctm.org/uploadedFiles/Research and Advocacy/research brief an

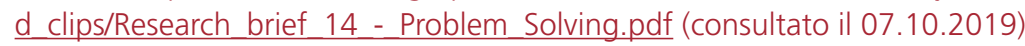

Pehkonen, E. (1993). What are Finnish teacher educators' conceptions about the teaching of problem solving in mathematics? European Journal for Teacher Education, 16(3), 237-256.

Pehkonen, E. (2017). Finnish elementary teachers' conceptions on problem solving in mathematics teaching, La Matematica e la sua Didattica, 25(1), 13-27.

Polya, G. (2016). Come risolvere i problemi di matematica. Utet Università Torino, 2016. (Titolo originale: How to solve it pubblicato nel 1945).

Richardson, V. (1996). The role of attitudes and beliefs in learning to teach. In J. Sikula, T. J. Buttery \& E. Guyton (Eds.), Handbook of Research on Teacher Education. A project of the Association of Teacher Educators (pp. 102-119). New York, NY: Macmillan Library.

Rokeach, M. (1960). The Open and Closed Mind: Investigations into the Nature of Belief Systems and Personality Systems. New York: Basic Books.

Schoenfeld, A. H. (1985). Mathematical problem solving. Academic Press, New York.

Schoenfeld, A. H. (1987). What's all the fuss about metacognition? In A. Schoenfeld (Ed.), Teaching and Learning Mathematical Problem Solving: Multiple Research Perspectives (pp. 189-215). Lawrence Erlbaum Associates, Hillsdale.

Schoenfeld, A. H. (1992). Learning to Think Mathematically: Problem Solving, Metacognition, and Sense Making in Mathematics. In D. A. Grows (Ed.), Handbook of Research on Mathematics Teaching and Learning (pp. 334-370). New York, NY: Macmillan. 
Schoenfeld, A. H. (2004). The math wars. Educational Policy, 18(1), 253-286.

Schön, D. A. (1983, trad.it. 1993). II professionista riflessivo. Per una nuova epistemologia della pratica. Bari: Dedalo.

Skott, J. (2009). Contextualising the notion of 'belief enactment'. Journal of Mathematics Teacher Education, 12(1), 27-46.

Tahan, M., Negrin, F., \& Zannini, L. (1996). L'uomo che sapeva contare: una raccolta di avventure matematiche. Salani.

Vannini, I. (2009). Ricerca empirico-sperimentale in Pedagogia: alcuni appunti su riflessione teorica e sistematicità metodologica. Ricerche di pedagogia e didattica, 4(1), 1000-1025.

Vannini, I. (2012). Come cambia la cultura degli insegnanti. Metodi per la ricerca empirica in educazione. Milano: Franco Angeli.

Vosniadou, S. (1994). Capturing and Modeling the Process of Conceptual Change. In S. Vosniadou (Ed.), Special Issue on Conceptual Change. Learning and Instruction, 4, 45-69.

Wilkins, J. (2008). The relationship among elementary teachers' content knowledge, attitudes, beliefs, and practices. Journal of Mathematics Teacher Education, 11(2), 139-164.

Xenofontos, C., \& Andrews, P. (2012). Prospective teachers' beliefs about problem-solving: Cypriot and English cultural constructions. Research in Mathematics Education, 14(1), 69-85.

Xenofontos, C., \& Andrews, P. (2014). Defining mathematical problems and problem solving: prospective primary teachers' beliefs in Cyprus and England, Mathematics Education Research Journal, 26(2), 279-299.

Zan, R. (2010). Difficoltà in matematica (osservare, interpretare, intervenire). Milano: Springer.

Zan, R. (2016). I problemi di matematica. Difficoltà di comprensione e formulazione del testo. Roma: Carocci Faber.

\section{Autore/Annarita Monaco}

NRD di Bologna, Università degli Studi di Roma "La Sapienza" - Italia

annarita.monaco@uniroma1.it 